\title{
Clinical feasibility of free-breathing, gadolinium-enhanced magnetic resonance angiography for assessing extracardiac thoracic vascular abnormalities in young children with congenital heart diseases
}

Sheung-Fat Ko, MD, ${ }^{a}$ Chi-Di Liang, MD, ${ }^{b}$ Chung-Cheng Huang, MD, ${ }^{a}$ Shu-Hang Ng, MD, ${ }^{a}$ Ming-Jang Hsieh, MD, ${ }^{c}$ Jen-Pin Chang, $\mathrm{MD}^{\mathrm{c}}$ and Min-Chi Chen, $\mathrm{PhD}^{\mathrm{d}}$

Supplemental material is available online.
From the Departments of Radiology, ${ }^{\text {a }}$ Pediatric Cardiology, ${ }^{\mathrm{b}}$ Thoracic and Cardiovascular, ${ }^{\mathrm{c}}$ and Public Health and Biostatistics, ${ }^{\mathrm{d}}$ Chang Gung University, Chang Gung Memorial Hospital at Kaohsiung, Kaohsiung Hsien, Taiwan.

Supported, in part, by grant no. NSC 892314-B-182A-114-M08 (to Dr Sheung-Fat Ko) from the National Science Council, Taiwan.

Received for publication March 7, 2006; revisions received April 22, 2006; accepted for publication May 8, 2006.

Address for reprints: Sheung-Fat Ko, MD, Department of Radiology, Chang Gung Memorial Hospital at Kaohsiung, 123 Ta-Pei Rd, Niao-Sung Hsiang, Kaohsiung Hsien 833, Taiwan (E-mail: sfa.ko@msa.hinet.net or sfatko@adm.cgmh.org.tw).

J Thorac Cardiovasc Surg 2006;132:1092-8 $0022-5223 / \$ 32.00$

Copyright (๑) 2006 by The American Association for Thoracic Surgery

doi:10.1016/j.jtcvs.2006.05.007
Objective: Congenital heart diseases in pediatric patients are associated with a wide variety of extracardiac thoracic vascular abnormalities. This study analyzed the value of gadolinium-enhanced magnetic resonance angiography during quiet free breathing for assessing extracardiac thoracic vascular abnormalities in young children with congenital heart diseases.

Methods: Fifty-three children with congenital heart diseases (age range, 1 day-40 months; mean, 10.9 months) associated with extracardiac thoracic vascular abnormalities who had undergone both free-breathing, gadolinium-enhanced magnetic resonance angiography and cardiac catheterization, surgical intervention, or both within 2 weeks were reviewed. Diagnostic findings on gadolinium-enhanced magnetic resonance angiography among patients grouped according to 3 major conditions of conotruncal abnormalities $(\mathrm{n}=33)$, aortic or venous abnormalities $(\mathrm{n}=$ $11)$, and pulmonary vascular abnormalities $(\mathrm{n}=9)$, as well as associated extracardiac thoracic vascular abnormality findings, were compared with findings made by using cardiac catheterization, surgical intervention, or both.

Results: Extracardiac thoracic vascular abnormality findings on gadoliniumenhanced magnetic resonance angiography were similar to those on catheterization, surgical intervention, or both in patients with conotruncal abnormalities (124 vs 127, $P=.083$ ), aortic or venous abnormalities (36 vs $33, P=.083$ ), and pulmonary vascular abnormalities (24 vs $25, P=.317$ ). The overall sensitivity of gadoliniumenhanced magnetic resonance angiography for detection of these lesions identified by means of catheterization, surgical intervention, or both was $97.9 \%$. However, gadolinium-enhanced magnetic resonance angiography revealed 11 additional extracardiac thoracic vascular abnormalities that were not found on cardiac catheterization. The $\kappa$ coefficient for the correlation of image quality and diagnostic value of gadolinium-enhanced magnetic resonance angiography by using a 4-point-scale $(1=$ nondiagnostic to $4=$ excellent) assessed by 2 independent reviewers was excellent (mean score $=3.66)$, with superb interobserver agreement $(\kappa=0.727-0.874)$.

Conclusions: Free-breathing, gadolinium-enhanced magnetic resonance angiography is clinically feasible for detailed anatomic delineation and treatment planning of various extracardiac thoracic vascular abnormalities in young children with congenital heart diseases.

$\mathrm{E}$ chocardiography and cardiac catheterization are among the mainstays of diagnosis of cardiovascular diseases. ${ }^{1-6}$ The low cost, noninvasiveness, and bedside portability of echocardiography make it ideally suited for use in infants and children. In many conditions echocardiography is the only imaging modal- 


\section{Abbreviations and Acronyms \\ CHD = congenital heart disease \\ ETVA = extracardiac thoracic vascular abnormality \\ GEMRA $=$ gadolinium-enhanced magnetic resonance angiography \\ MAPCA $=$ major aortopulmonary collateral artery \\ PAPVR = partial anomalous pulmonary venous return \\ $\mathrm{SVC}=$ superior vena cava \\ TAPVR $=$ total anomalous pulmonary venous return \\ TGA $=$ transposition of the great arteries \\ $\mathrm{ToF}=$ tetralogy of Fallot}

findings with those of cardiac catheterization, surgical intervention, or both.

\section{Methods \\ Patients}

All children less than 4 years of age referred to our department for cardiovascular magnetic resonance examination from January 1, 2000 , to June 30 , 2005, who fulfilled the following criteria were included in this study: (1) diagnosis of CHD with cardiovascular defects (atrial or ventricular septal defect, patent ductus arteriosus, abnormal cardiac chambers, and/or valvular abnormalities) that required interventional or surgical treatment; (2) suspected association with ETVAs (conotruncal, aortic, central venous, and/or pulmonary arterial or venous lesions); (3) underwent quiet freebreathing GEMRA; and (4) underwent cardiac catheterization, surgical confirmation, or both within 2 weeks of the GEMRA study. All GEMRA studies were clinically indicated for detailed anatomic delineation of ETVAs. This retrospective study was approved by the institutional review board of our hospital, including the use of data obtained by searching of computer databases and the review of medical records.

\section{Three-dimensional GEMRA}

Informed parental consent for GEMRA was obtained, and all examinations were performed with a 1.5 -T superconducting magnetic resonance system (Signa Horizon LX EchoSpeed 8.1/8.3 Operating System; GE Healthcare, Milwaukee, Wis). Intravenous access was established with a 22- or 24-gauge intravenous cannula inserted into a peripheral vein. Light anesthesia was routinely applied by means of administration of oral choral hydrate (75 $\mathrm{mg} / \mathrm{kg}$, maximal dose was $2000 \mathrm{mg}$ ). If not successful, intravenous ketamine $(1 \mathrm{mg} / \mathrm{kg})$ and atropine $(0.01 \mathrm{mg} / \mathrm{kg})$ were administered. The oxygen saturation and heart rate of the lightly sedated patients were continuously monitored with a peripheral gating clamp fixed at the thumb or middle finger. A head coil was used in patients weighing less than or equal to $10 \mathrm{~kg}$. A cardiac phase-array coil was used in those weighing greater than $10 \mathrm{~kg}$. Localizing images were obtained in the coronal plane with the center at the midthorax by using a fast spoiled-gradient echo-recall sequence. Afterward, free-breathing GEMRA was performed with a nonelectrocardiogramtriggered, 3-dimensional, spoiled gradient-recalled echocardiographic pulse sequence with the following parameters: flip angles of $45^{\circ}$; repetition time of 5.5 to $7.8 \mathrm{~ms}$; echocardiographic time of 1.2 to $2.5 \mathrm{~ms}$; number of excitations of 1 ; rectangular field of view with maximum dimension of 16 to $28 \mathrm{~cm}$ depending on body size; $256 \times 128$ matrix; centric k-space filling; sampling bandwidth of $31.2 \mathrm{kHz}$; slab thickness of 8 to $12 \mathrm{~cm}$ with 50 to 70 partitions, effective slice thickness of 1.5 to $2.2 \mathrm{~mm}$; and acquisition time of 20 to 45 seconds. Gadopentate dimeglumine $(0.2 \mathrm{mmol} / \mathrm{kg}$ body weight; Magnevist, Schering, Berlin, Germany) was administered by means of manual injection through the peripheral cannula and followed by saline flushing. Because of the presence of CHDs with intracardiac or extracardiac shunts, as well as fast heart rates in small children, the "best guess" method with a delay time of 6 to 8 seconds was used. ${ }^{14,20,21}$ Three uninterrupted sequential acquisitions were performed with quiet breathing.

After the examination, the raw data were immediately transferred to a commercially available workstation (Advantage Work- 
station, AW 4.0, GE Healthcare). Postprocessing of GEMRA with multiplanar reconstructions, 2-dimensional composite and subvolume maximal intensity projections, and 3-dimensional projection angiograms created with surface-shaded rendering techniques to best depict particular anatomic features of interest was used. Userdefined subvolume maximal intensity projection images were used to assess the extent of stenosis. Data segmentation methods were used to remove unnecessary overlapping structures so that the regions of interest were clearly shown. Two radiologists blinded to the clinical status of the patients were asked to reformat and analyze the images independently. Disagreements were resolved by consensus. The time devoted to the analysis of each case was approximately 30 minutes.

The ETVAs were divided into 3 main categories: (1) conotruncal developmental abnormalities; (2) aortic or central venous developmental abnormalities; and (3) pulmonary vascular developmental abnormalities. For conotruncal lesions, the following anatomic abnormalities were investigated: findings compatible with dextro- or levo-transposition of the great arteries (D-TGA or L-TGA); tetralogy of Fallot (ToF), the status of pulmonary arteries and confluence, each individual major aortopulmonary collateral artery (MAPCA; $\geq 2 \mathrm{~mm}$ in diameter), or the presence of clustered multiple small aortopulmonary collateral arteries (each with diameter $<2 \mathrm{~mm}$ ) and abnormal operative shunts; interrupted aortic arch; right aortic arch; double-outlet right ventricle; truncus arteriosus; and aortopulmonary window or fenestration. For aortic or central venous lesions, the following anatomic abnormalities were investigated: findings compatible with coarctation of the aorta and collaterals; anomalous aortic branching (eg, aberrant right or left subclavian artery, retroesophageal aortic diverticulum, and mirror-image branching); or central venous lesions (eg, abnormal superior vena cava [SVC] or inferior vena cava and abnormal azygos or hemiazygos veins). For pulmonary vascular abnormalities, the following anatomic abnormalities were investigated: findings compatible with main, right, or left pulmonary artery atresia or stenosis; the absence of pulmonary arterial confluence, and partial anomalous pulmonary venous return (PAPVR) or total anomalous pulmonary venous return (TAPVR). In each case abnormal widening, narrowing, or occlusion of the vessels or shunts and other associated anomalous vascular structures were also recorded.

\section{Cardiac Catheterization/Surgical Findings}

Cardiac catheterization examinations were performed by experienced pediatric cardiologists using a BH 5000 system (Philips Medical Systems, Best, The Netherlands). Aortography, ventriculography, and study of pulmonary arteries were performed routinely. Wedged pulmonary venography and selective catheterization of the carotid, subclavian, and MAPCA arteries were performed as needed. A pediatric cardiologist who was unaware of the magnetic resonance angiography findings reviewed the cardiac catheterization images, and the number of ETVAs was determined by using a similar methodology as for GEMRA. For those patients who did not undergo surgical correction, GEMRA was compared with cardiac catheterization findings. For surgical patients (without or with prior cardiac catheterization studies), GEMRA was primarily compared with surgical observations, and the surgical records were jointly reviewed by a radiologist and a cardiovascular surgeon.

\section{Statistical Analysis}

The number of ETVAs, including the anatomic variables described above in each category, and the associated vascular lesions revealed on GEMRA in each patient were compared with the findings on cardiac catheterization, surgical intervention, or both by using the nonparametric paired Wilcoxon signed-rank test.

\section{Assessment of Imaging Quality and Clinical Feasibility}

The assessment of the overall quality of 3-dimensional GEMRA (including the presence or absence of motion artifact, the continuity of the vessels, and the sharpness and demarcation of the vascular outline) and clinical value were subjectively graded on a 4-point-scale as follows: 1, poor quality and inadequate information for answering clinical queries and making decisions about further treatment; 2 , fair quality but with inadequate information for answering clinical queries and making decisions about further treatment; 3, good quality and adequate information for answering clinical queries and making decisions about further treatment; and 4 , excellent quality with adequate information for answering clinical queries and making decisions about further treatment. The GEMRA-reformatted images were rated by a cardiovascular surgeon and then independently rated by a radiologist who did not perform the image processing. The interobserver agreement of the quality scores was evaluated with the $\kappa$ coefficient. The degree of concordance was considered to be "poor" when the $\kappa$ value was less than 0.2 , "fair" when the $\kappa$ value was 0.2 or greater to less than 0.4 , "moderate" when the $\kappa$ value was 0.4 or greater to less than 0.6 , "good" when the $\kappa$ was 0.6 or greater to less than 0.8 , and "excellent" when the $\kappa$ value was 0.8 or greater to 1.0 .

\section{Results}

A total of 53 patients (20 female and 33 male patients; age range, 1 day-40 months; mean, 10.9 months) met the inclusion criteria for the study. The major diagnoses among patients in the conotruncal developmental, aortic or venous developmental, and pulmonary vascular abnormality groups and their associated ETVAs are summarized in Tables E1 through E3. Conotruncal abnormalities were diagnosed in 33 patients (11 with D-TGA or L-TGA, 13 with ToF, 2 with interrupted aortic arch, 2 with right aortic arch, 1 with double-outlet right ventricle, 2 with truncus arteriosus, 1 with aortopulmonary fenestration, and 1 with aortopulmonary window), aortic or venous developmental abnormalities were diagnosed in 11 patients ( 7 with coarctation of the aorta, 2 with anomalous aortic branches, 1 with a double SVC and the persistent left SVC draining to a dilated coronary sinus, and 1 with left isomerism), and pulmonary arterial or venous abnormalities were diagnosed in 9 patients ( 6 with pulmonary atresia or stenosis, 1 with widened pulmonary artery and pulmonary valve dysplasia, 1 with PAPVR, and 1 with TAPVR). In 44 patients GEMRA was accomplished by using oral choral hydrate only. However, inadequate sedation with choral hydrate led to aborted studies in 9 patients, and successful repeated studies were achieved on the next day by using intravenous ketamine. No conversion to general anesthesia was needed. None of the 
patients had any sedation-related complications, and neither additional respiratory nor inotropic support was required.

\section{Detection of Associated Extracardiac Vascular Abnormalities}

The numbers of extracardiac vascular abnormalities found on GEMRA, cardiac catheterization, and/or surgical intervention are summarized in Tables E1 through E3. There was no significant difference in the detection of abnormalities on GEMRA versus catheterization, surgical intervention, or both in the conotruncal abnormalities group (124 vs $127, P=.083$ ), aortic or venous abnormalities group (36 vs $33, P=.083$ ), and pulmonary abnormalities group (24 vs 25 , $P=.317)$.

In the conotruncal abnormalities group 18 of 33 patients underwent both cardiac catheterization and surgical intervention. Among these 18 patients, except for multiple small MAPCAs that were not visible on GEMRA in 2 patients, GEMRA revealed all abnormalities diagnosed on catheterization in the other 16 patients, as well as 7 additional abnormalities (1 pulmonary stenosis, 3 MAPCAs, 1 TAPVR, 1 PAPVR, and 1 aberrant right subclavian artery) that were all confirmed on subsequent operations. In the remaining 15 patients, GEMRA also revealed all vascular abnormalities seen on catheterization or surgical intervention (Figure E1), except for one mild supravalvular pulmonary stenosis in a patients with D-TGA postaortopulmonary switch.

In the aortic or venous abnormalities group, only 3 of 11 patients underwent both cardiac catheterization and surgical intervention, and GEMRA revealed all abnormalities found on catheterization and surgical intervention. In the remaining 8 patients, GEMRA also revealed all vascular abnormalities seen on catheterization, as well as 3 additional abnormalities ( 1 aberrant left subclavian artery, 1 subclavian steal caused by severe innominate stenosis, and 1 hemiazygal continuation to persistent left SVC; Figure 1).

In the pulmonary vascular abnormalities group, only 2 of 9 patients underwent both cardiac catheterization and surgical intervention, whereas pulmonary valve dysplasia in 1 patent was not visible on GEMRA. Among the remaining 7 patients (4 underwent catheterization only, and 3 underwent surgical intervention directly after GEMRA), all lesions found on catheterization or surgical intervention were accurately revealed on GEMRA (Figure E2).

Compared with the number of abnormalities revealed on cardiac catheterization, surgical intervention, or both, GEMRA exhibited $97.6 \%, 100 \%$, and $96 \%$ sensitivities in detection of conotruncal, aortic or venous, and pulmonary vascular abnormalities, respectively, with an overall sensitivity of $97.9 \%$. Furthermore, 11 additional ETVAs were revealed on GEMRA that were not found on cardiac catheterization.

\section{Image Quality and Clinical Feasibility}

GEMRA was considered diagnostic in all 53 patients with good to excellent quality and mean quality scores for conotruncal, aortic or venous, and pulmonary vascular abnormalities of 3.60, 3.75, and 3.71, respectively. Excellent interobserver correlations of quality scores for conotruncal abnormalities $(\kappa=0.874, P<.001)$, aortic or venous abnormalities $(\kappa=0.744, P=.011)$, and pulmonary vascular abnormalities $(\kappa=0.727, P=.023)$ were found between the 2 raters. These results validated the clinical feasibility of GEMRA during light anesthesia and free breathing in the assessment of various ETVAs in neonates, infants, and young children with CHDs.

\section{Discussion}

Spin-echocardiographic and gradient-recalled magnetic resonance sequences can offer clear demonstration of thoracic vascular abnormalities but are limited by long scanning time, the requirement for multiple breath holds, low spatial resolution, turbulent flow, slow flow, and underlying conditions, such as arrhythmia, severe pulmonary disease, and poor cooperation. ${ }^{1-6,8-10,22,23}$ GEMRA has shown great promise in the evaluation of thoracic vessels, even for patients with low cardiac output or severely altered hemodynamics, by virtue of rapid T1 shortening of blood with paramagnetic agents (gadolinium) by considerable reduction of acquisition times and high signal-to-noise ratio, preferential enhancement of arteries, elimination of turbulent artifacts, larger volume of acquisition, and multiplanar capabilities. ${ }^{10-17}$

GEMRA is highly accurate in the evaluation of aortic coarctation, complex pulmonary arterial stenosis and atresia, systemic and pulmonary venous anomalies, and MAPCAs. Most studies of GEMRA have emphasized breath holding during the examination. ${ }^{10-17}$ In pediatric patients, however, especially in neonates or infants, GEMRA is typically conducted during general anesthesia, with ventilation suspended during imaging. ${ }^{12-18}$ Anesthetizing pediatric patients with CHDs for cardiac magnetic resonance imaging poses many challenges for the anesthesiologist. Although complications are rare, general anesthesia carries potential risks, especially in small children, because a longer connecting tube from the patient to the ventilator within the magnetic resonance gantry is required. In addition, low cardiac output, necessity for administration of an inotropic agent after anesthesia induction, and transient hypotension might be encountered. ${ }^{18}$ Furthermore, the cost entailed for general anesthesia for magnetic resonance imaging is much higher than for free-breathing light anesthesia (approximately $\$ 350-\$ 450$ vs $\$ 10-\$ 20$ in our hospital). GEMRA during spontaneous breathing with inhalation of sevoflurane for anesthesia of children has been reported. ${ }^{11}$ In this study GEMRA was performed during free-breathing light anes- 

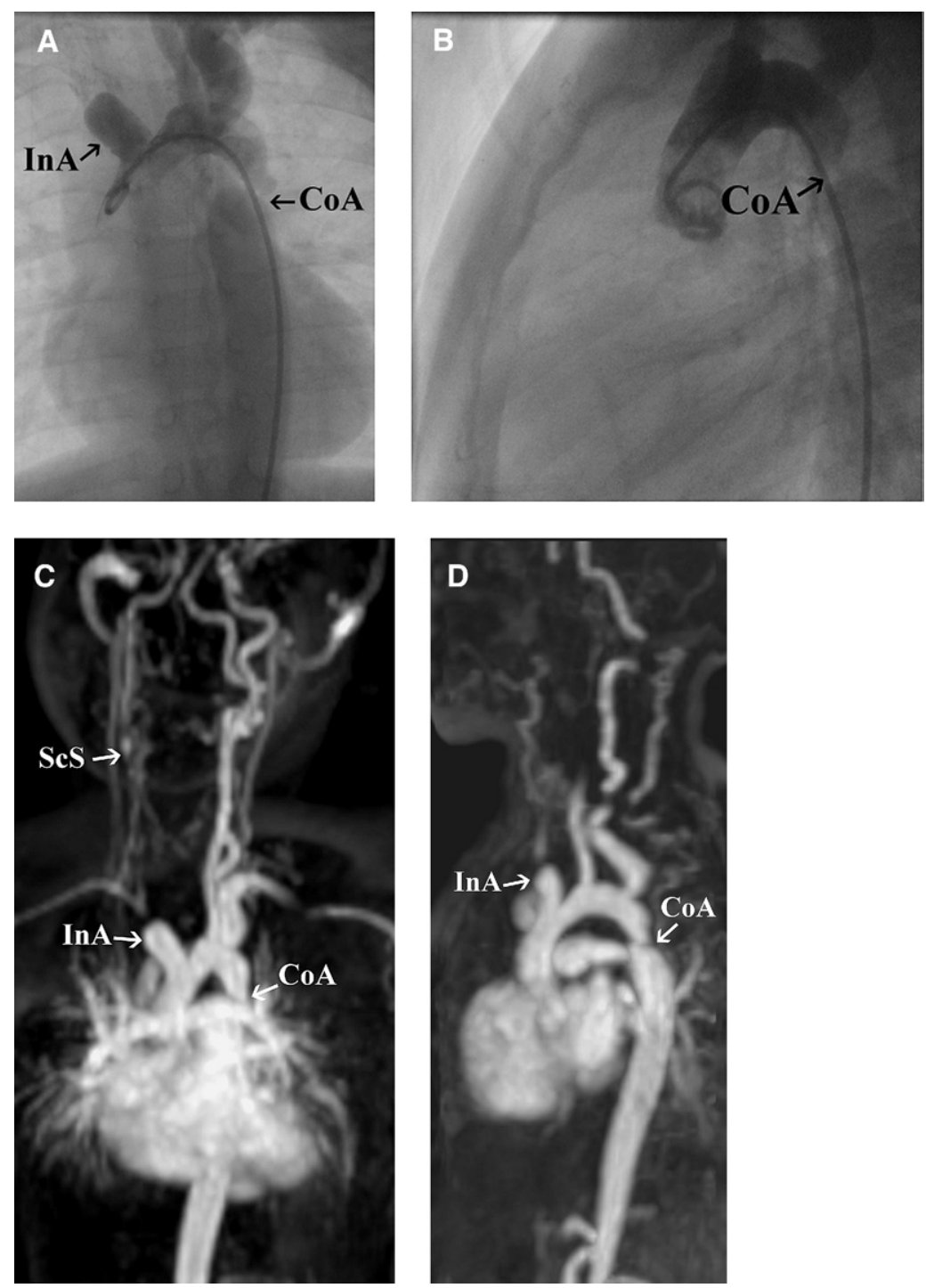

Figure 1. A 28-month-old boy with angiographically proved segmental aortic coarctation associated with right innominate artery occlusion and subclavian steal phenomenon. A and B, Coronal and sagittal aortograms show aortic coarctation (CoA) just beyond the dilated left subclavian aorta and the dilated proximal right innominate artery $(\operatorname{InA})$ with abrupt occlusion. $C$ and D, GEMRA in coronal and sagittal subvolume maximal intensity projection images clearly reveal coarctation (COA) and prominent collateral arterial supplies from the descending aorta. Note occlusion of the right innominate artery $(\operatorname{In} A)$ with proximal dilatation and elucidation of the subclavian steal phenomenon $(S c S)$, which was not seen on aortograms. thesia by using oral choral hydrate first and, if not successful, subsequent intravenous administration of ketamine. The examinations were successfully performed in all patients without any complications. The results showed that freebreathing GEMRA is also a clinically feasible method with high sensitivity (97.9\%) for the evaluation of ETVAs and no significant difference in findings compared with catheterization, surgical intervention, or both. However, for young children with CHDs who are ventilator dependent or have severe congestive heart failure or marked pulmonary hypertension and in whom intravenous administration of ketamine and atropine is not appropriate, free-breathing GEMRA is hindered.

Conotruncal abnormalities are related to abnormal division or rotation of the primitive truncus during embryologic development. Standard spin-echocardiographic and gradi- ent-echocardiographic techniques can effectively depict the anatomic relationship between the aorta and the pulmonary artery. $^{24}$ This study has demonstrated that GEMRA is highly accurate in the diagnosis of various conotruncal abnormalities, including D-TGA or L-TGA, ToF, interrupted aorta, right aortic arch, double-outlet right ventricle, truncus arteriosus, aortopulmonary window, and even small aortopulmonary fenestration in young children. Pulmonary stenoses and associated aortic, venous, and pulmonary ETVAs were clearly shown by GEMRA in all of our patients with TGA, except in one who had received an arterial switch procedure in another hospital and had a mild supravalvular pulmonary stenosis, which was overlooked. The importance of multiplanar assessment of GEMRA for follow-up examinations of TGA after arterial switch has been stressed because pulmonary artery stenoses are usually 
ovoid shaped and might erroneously be interpreted as normal in some views. ${ }^{25}$

Previous studies have shown that GEMRA is a robust method, with $100 \%$ sensitivity for delineation of all sources of pulmonary blood supplies in patients with complex pulmonary stenosis and atresia, such as ToF, as well as various pulmonary and systemic venous anomalies. ${ }^{12-15}$ Prasad and associates $^{12}$ reported that all MAPCAs with diameters of $2.5 \mathrm{~mm}$ or greater and clusters of smaller MAPCAs were diagnosed on GEMRA, and results showed excellent correlation with cardiac catheterization. Geva and colleagues ${ }^{14}$ and Greil and coworkers ${ }^{13}$ reported that the good signal-tonoise ratio of GEMRA results in high-resolution demonstration of small vessels down to $0.5 \mathrm{~mm}$ in diameter. Excellent correlation between GEMRA and angiographic measurements of congenital stenoses in small pulmonary arteries has also been reported. ${ }^{15}$ In this study GEMRA performed during light anesthesia in young children identified all MAPCAs with a diameter of $2 \mathrm{~mm}$ or greater. Although small clusters of MAPCAs were not visible in 2 cases in the present study, this did not affect the treatment or outcome because the affected vessels were too small and had slow blood flow. On the other hand, GEMRA is helpful by providing an anatomic road map for coil embolization of high-flow MAPCAs, which might generate pulmonary overcirculation. ${ }^{13,14}$

This study found that even for children less than 40 months old, GEMRA was 100\% sensitive in differentiation of segmental narrowing or tubular hypoplasia of aortic coarctation, evaluation of collateral supplies, and depiction of associated anomalies, including Kommerell's diverticulum, aberrant subclavian artery, and mirror-image branching. Moreover, a total of 3 uninterrupted sequential acquisitions were routinely obtained from our patients, allowing comprehensive investigation of venous abnormalities, such as persistent left SVC, interrupted IVC with hemiazygos continuation, and unusual delayed arterial perfusion with subclavian steal phenomenon. GEMRA also provides accurate quantitative anatomic information of the aorta in children with CHDs, which is well correlated with conventional angiographic data and can therefore be used for planning catheter-guided procedures. ${ }^{16}$ GEMRA has the advantage of being a radiation-free tool for follow-up of patients with aortic coarctation after transcatheter dilatation or surgical repair, who are expected to require lifelong surveillance, and this might be especially important for those who undergo repair in infancy and young childhood and are thus more likely to have recurrent coarctation or aneurysm formation. ${ }^{17}$

Three-dimensional GEMRA data sets should be acquired to match to k-space reconstruction and to synchronize peak contrast concentration in the target vessels during data acquisition to obtain optimal vascular enhancement. ${ }^{15,26,27} \mathrm{~A}$ contrast dose of $0.2 \mathrm{mmol} / \mathrm{kg}$ body weight was routinely used in this study to obtain a high signal-to-noise ratio, even during free breathing. ${ }^{19}$ For image acquisition, automated triggering with bolus tracking or timing with a time-intensity curve after a test bolus was not used in this study. Fast heart rates, as well as the presence of various complex intracardiac and extracardiac shunts, limited the application of such triggering methods in this cohort of young children, necessitating the use of only a small amount of contrast agent. Circulation times determined during end-inspiration breath hold might differ from those obtained during free breathing by as much as 6 seconds in an individual patient. ${ }^{27}$ The "best guess" method with initiation of the acquisition approximately 6 to 8 seconds after manual injection of contrast medium injection can provide high-quality GEMRA images. ${ }^{14,20,21}$ The $\kappa$ coefficient for correlation of the image quality and diagnostic value of GEMRA during light anesthesia, as assessed by 2 independent reviewers, in this study revealed excellent image quality (mean score $=3.66$ ) with superb interobserver agreement $(\kappa=0.727-0.874)$. This finding validates the feasibility of this method for evaluation of a wide spectrum of ETVAs in young children with CHDs.

\section{Limitations}

This study had several limitations. Not all patients underwent both cardiac catheterization and surgical intervention, which would not have been practical and ethical in some patients who could be adequately managed with a single interventional treatment. On the other hand, some of our patients underwent operations directly after GEMRA, which served as an excellent tool for surgical planning. The sample size was small in each of the 3 major diagnostic groups in this study, and the spectrum of ETVAs in these patients was wide. However, the inclusion criteria required cardiac catheterization, surgical intervention, or both in all selected cases so that GEMRA findings could confidently be confirmed. The quality score and clinical feasibility of GEMRA rated by a cardiovascular surgeon and a radiologist in this study were based on the GEMRA-reformatted images, and these evaluators did not perform postprocessing image reconstructions. Although the GEMRA findings might have been influenced by the radiologist who performed the image reconstructions, this method was necessary to ensure that these evaluators were truly blinded to the image findings before assessment.

\section{Conclusions}

Free-breathing GEMRA is clinically feasible for comprehensive anatomic delineation of a wide spectrum of ETVAs in young children with CHDs, facilitating transcatheter or surgical treatment. 


\section{References}

1. Krabill KA, Ring WS, Foker JE, Braunlin EA, Einzig S, Berry JM, et al. Echocardiography versus cardiac catheterization diagnosis of infants with congenital heart disease requiring cardiac surgery. Am J Cardiol. 1987;60:351-4.

2. Dorfman AL, Levine JC, Colan SD, Geva T. Accuracy of echocardiography in low birth weight infants with congenital heart disease. Pediatrics. 2005;115:102-7.

3. Geva T, Kreuter J. Diagnostic pathways for evaluation of congenital heat disease. In: Crawford MH, DiMarco JP, editors. Cardiology. London: Mosby International; 2001. p. 1-22.

4. Marx GR, Geva T. MRI and echocardiography in children: how do they compare? Semin Roentgenol. 1998;33:281-92.

5. Vick GW, Rokey R, Huhta JC, Mulvagn SL, Johnstone DL. Nuclear magnetic resonance imaging of the pulmonary arteries, subpulmonary region and aorticopulmonary shunts: a comparative study with twodimensional echocardiography and angiography. Am Heart J. 1990; 119:1103-10

6. Stanger P, Heymann MA, Tarnoff H, Hoffman JIE, Rudolph AM. Complications of cardiac catheterization of neonates, infants and children. Circulation. 1974;50:595-608.

7. Vitiello R, McCrindle BW, Nykanen D, Freedom RM, Benson LN. Complications associated with pediatric cardiac catheterization. $J$ Am Coll Cardiol. 1998;32:1433-40.

8. Prince MR, Narasimham DL, Jacoby WT, Williams DM, Cho KJ, Marx MV, et al. Three-dimensional gadolinium enhanced MR angiography of the thoracic aorta. AJR Am J Roentgenol. 1996;166:1387-97.

9. Krinsky GA, Rofsky NM, DeCorato DR, Weinreb JC, Earls JP, Flyer MA, et al. Thoracic aorta: comparison of gadolinium-enhanced threedimensional MR angiography with conventional MR imaging. Radiology. 1997;202:183-93.

10. Ho VB, Prince MR. Thoracic MR aortography: imaging techniques and strategies. Radiographics. 1998;18:287-309.

11. Caseiro-Alves D, Gil-Agnostinho P, Ramalheiro G, Gil-Agnostinho P. Contrast-enhanced MR angiography of thoracic vascular malformations in a pediatric population. AJR Am J Roentgenol. 2003;181:861-6.

12. Prasad SK, Soukias N, Hornung T, Khan M, Pennell DJ, Gatzoulis MA, et al. Role of magnetic resonance angiography in the diagnosis of major aortopulmonary collateral arteries and partial anomalous pulmonary venous drainage. Circulation. 2004;109:207-14.

13. Greil GF, Powell AJ, Gildein HP, Geva T. Gadolinium-enhanced 3-dimensional magnetic resonance angiography of pulmonary and systemic venous anomalies. J Am Coll Cardiol. 2002;39:335-41.

14. Geva T, Greil GF, Marshall AC, Landzberg M, Powell AJ. Gadoliniumenhanced 3-dimensional magnetic resonance angiography of pulmonary blood supply in patients with complex pulmonary stenosis or atresia: comparison with $\mathrm{x}$-ray angiography. Circulation 2002;106:473-8

15. Kondo C, Takada K, Yokoyama U, Nakajima Y, Momma K, Sakai F. Comparison of three-dimensional contrast-enhanced magnetic resonance angiography and axial radiographic angiography for diagnosing congenital stenoses in small pulmonary arteries. Am J Cardiol. 2001; 87:420-4.

16. Valsangiacomo Buchel ER, Dibernardo S, Bauersfeld U, Berger F. Contrast-enhanced magnetic resonance angiography of the great arteries in patients with congenital heart disease: an accurate tool for planning catheter-guided interventions. Int $J$ Cardiovasc Imaging. 2005;21:313-22.

17. Konen E, Merchant N, Provost Y, McLaughlin PR, Crossin J, Paul NS Coarctation of the aorta before and after correction: the role of cardiovascular MRI. AJR Am J Roentgenol. 2004;182:1333-9.

18. Odegard KC, DiNardo JA, Tsai-Goodman B, Powell AJ, Geva T, Laussen PC. Anaesthesia considerations for cardiac MRI in infants and small children. Paediatr Anaesth. 2004;14:471-6.

19. Holmqvist C, Larsson E-M, Stahlberg F, Laurin S. Contrast-enhanced thoracic 3D-MR angiography in infants and children. Acta Radiol. 2001;42:50-8.

20. Macgowan CK, Al-Kwifi O, Varodayan F, Yoo SJ, Wright GA, Kellenberger CJ. Optimization of 3D contrast-enhanced pulmonary magnetic resonance angiography in pediatric patients with congenital heart disease. Magn Reson Med. 2005;54:207-12.

21. Prince MR. Contrast-enhanced MR angiography: theory and optimization. Magn Reson Imaging Clin N Am. 1998;6:257-67.

22. Nienaber CA, Spielmann RP, von Kodolitsch Y, Siglow S, Piepho A, Juap T, et al. Diagnosis of thoracic aortic dissection: Magnetic resonance imaging versus transesophageal echocardiography. Circulation. 1991;85:434-47.

23. Hernandez RJ. Cardiovascular MR imaging of children. Magn Reson Imaging Clin N Am. 1996;4:615-35.

24. Donnelly LF, Higgins CB. MR imaging of conotruncal abnormalities AJR Am J Roentgenol. 1996;166:925-8.

25. Weiss F, Habermann CR, Lilje C, Nimz M, Rasek V, Dallmeyer J, et al. MRI of pulmonary arteries in follow-up after arterial-switchoperation (ASO) for transposition of great arteries (d-TGA). Rofo. 2005; 177:849-55.

26. Wang Y, Winchester PA, Yu L, Watts R, Ding G, Lee HM, et al Breath-hold three-dimensional contrast-enhanced coronary MR angiography: motion-matched $\mathrm{k}$-space sampling for reducing cardiac motion effects. Radiology. 2000;215:600-7.

27. Krinsky GA, Kaminer E, Lee VS, Rofsky NM, Weinreb JC. The effects of apnea on timing examinations for optimization of gadoliniumenhanced MRA of the thoracic aorta and arch vessels. J Comput Assist Tomogr. 1998;22:677-81. 


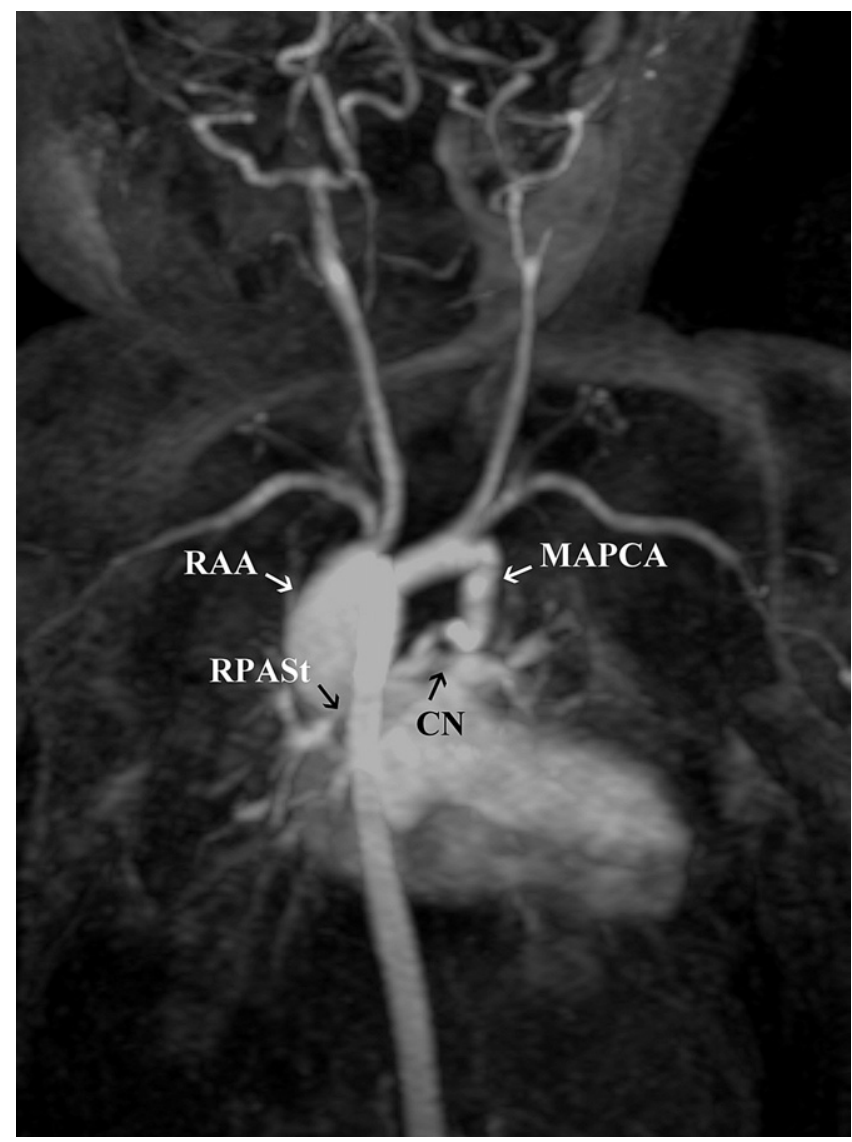

Figure E1. A 5-day-old boy with tetralogy of Fallot underwent surgical intervention directly after gadolinium-enhanced magnetic resonance angiography. Gadolinium-enhanced magnetic resonance angiography in coronal subvolume maximal intensity projection image reveals the right aortic arch (RAA) from a major aortopulmonary collateral artery (MAPCA), which runs a tortuous course and then connects to the proximal part of the right pulmonary artery (RPA). Note mild confluence narrowing (CN) and distal RPA stenosis (RPASt).

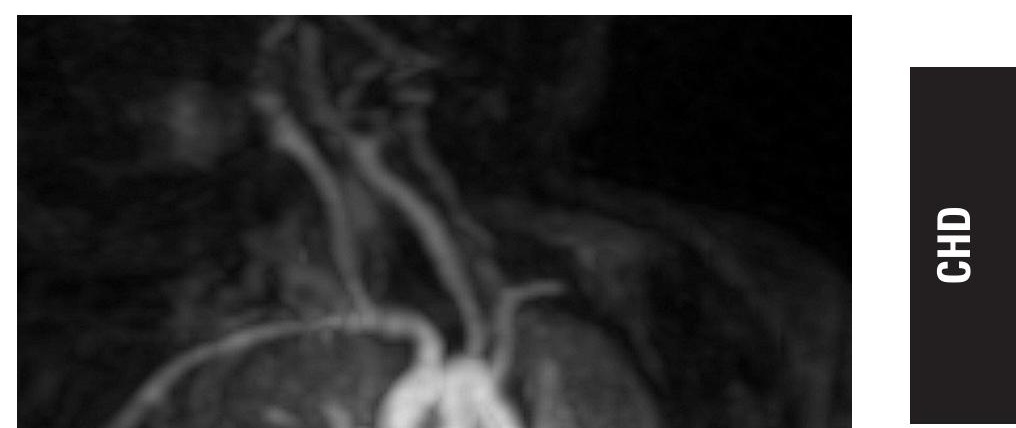

Figure E2. A 4-day-old girl with infradiaphragmatic total anomalous pulmonary venous return (TAPVR) underwent surgical intervention directly after gadolinium-enhanced magnetic resonance angiography. Gadolinium-enhanced magnetic resonance angiography in coronal subvolume maximal intensity projection image reveals that the pulmonary veins $(P V)$ join into a common trunk (TAPVR), which subsequently drains into the portal vein (PoV). $R U P V$, right upper pulmonary vein; $R L P V$, right lower pulmonary vein; $L L P V$, left lower pulmonary vein. 
TABLE E1. Summary of conotruncal developmental abnormalities and associated vascular abnormalities in 33 young patients with congenital heart diseases

\begin{tabular}{|c|c|c|c|c|c|c|}
\hline Case no. & Age/sex & Major diagnosis & Associated findings & Prior procedures & MRA & $\begin{array}{l}\text { Catheterization/ } \\
\text { operation }\end{array}$ \\
\hline \multicolumn{7}{|c|}{ Transposition of the great arteries } \\
\hline 2 & $3 \mathrm{mo} / \mathrm{F}$ & D-TGA & PS, PDA & & 3 & $2 / 3$ \\
\hline 3 & $5 \mathrm{mo} / \mathrm{F}$ & D-TGA & PS, RAA & & 3 & $3 / \mathrm{N}$ \\
\hline 6 & $11 \mathrm{mo} / \mathrm{F}$ & D-TGA & PS & BTS & 2 & $2 / 2$ \\
\hline 7 & $23 \mathrm{mo} / \mathrm{F}$ & D-TGA after switch & PS & AP switch & 1 & $2 / \mathrm{N}$ \\
\hline 8 & $3 \mathrm{~d} / \mathrm{M}$ & D-TGA & Widened MPA, PDA & & 3 & $3 / \mathrm{N}$ \\
\hline 9 & $2 \mathrm{mo} / \mathrm{M}$ & D-TGA & $\begin{array}{l}\text { Widened MPA, CoA with } \\
\text { hypoplastic arch, ARSA }\end{array}$ & & 4 & $4 / 4$ \\
\hline 12 & $2 \mathrm{mo} / \mathrm{F}$ & ToF & $\begin{array}{l}\text { PAt, C+, CN, MAPCAs (2), RAA, } \\
\text { RPA stenosis, DSVC }\end{array}$ & & 7 & $\mathrm{~N} / 7$ \\
\hline 13 & $1 \mathrm{~d} / \mathrm{M}$ & ToF & PAt, C+, MAPCAs (2) & & 3 & $3 / \mathrm{N}$ \\
\hline 14 & $5 \mathrm{~d} / \mathrm{M}$ & ToF & $\begin{array}{l}\text { PAt, C+, CN, RPA stenosis, } \\
\text { RAA, MAPCA (1) }\end{array}$ & & 5 & $\mathrm{~N} / 5$ \\
\hline 15 & $25 \mathrm{mo} / \mathrm{M}$ & ToF & PAt, C+, CN, MAPCAs (2) & BTS & 4 & $4 / 4$ \\
\hline 16 & $4 \mathrm{mo} / \mathrm{M}$ & ToF & PAt, C-, MAPCAs (3) & & 5 & $4 / 5$ \\
\hline 17 & $11 \mathrm{mo} / \mathrm{F}$ & ToF & PAt, C-, MAPCAs $(3+\mathrm{Mu})$ & BTS & 6 & $5 / 6$ \\
\hline 18 & $7 \mathrm{mo} / \mathrm{F}$ & ToF & PAt, C-, MAPCAs (2) & BTS & 4 & $4 / 4$ \\
\hline 19 & $24 \mathrm{mo} / \mathrm{M}$ & ToF & PAt, $\mathrm{C}-$, shunt aneurysm, PDA & Waterston shunt & 4 & $4 / 4$ \\
\hline 20 & $38 \mathrm{mo} / \mathrm{M}$ & ToF & $\begin{array}{l}\text { PAt, } \mathrm{C}-\text {, conduit stenosis, RPA } \\
\text { aneurysm, MAPCA }(1+\mathrm{Mu})\end{array}$ & Rastelli procedure & 5 & $6 / 6$ \\
\hline \multicolumn{7}{|l|}{ Others } \\
\hline 25 & $1 \mathrm{mo} / \mathrm{F}$ & Interrupted aortic arch & Numerous collateral vessels & & 2 & $2 / \mathrm{N}$ \\
\hline 26 & $3 \mathrm{~d} / \mathrm{M}$ & Interrupted aortic arch & $\begin{array}{l}\text { Descending aorta from PDA, left } \\
\text { innominate artery }\end{array}$ & & 3 & $\mathrm{~N} / 3$ \\
\hline 27 & $2 \mathrm{wk} / \mathrm{F}$ & RAA, RAD, ALSA & & & 3 & $3 / \mathrm{N}$ \\
\hline 28 & 3 wk/M & RAA, mirror-image branching & & & 3 & $\mathrm{~N} / 3$ \\
\hline 29 & $18 \mathrm{mo} / \mathrm{F}$ & Double-outlet right ventricle & $\begin{array}{l}\text { IVC agenesis, azygos and } \\
\text { hemiazygos continuation }\end{array}$ & & 4 & $4 / \mathrm{N}$ \\
\hline 30 & 2 wk/F & Type 2 truncus & PDA replaced arch, ARSA & & 3 & $3 / 3$ \\
\hline 31 & $39 \mathrm{mo} / \mathrm{M}$ & Type 4 truncus & RAA, RPA aneurysm & & 3 & $3 / \mathrm{N}$ \\
\hline 32 & $9 \mathrm{mo} / \mathrm{M}$ & AP fenestration & & & 1 & $1 / 1$ \\
\hline 33 & $5 \mathrm{~d} / \mathrm{M}$ & AP window & ARSA & & 2 & $1 / 2$ \\
\hline
\end{tabular}

$M R A$, Number of abnormalities seen on magnetic resonance angiography; Catheterization/operation, number of abnormalities seen on cardiac catheterization, surgical intervention, or both; D-TGA, dexo-transposition of great arteries; $P S$, pulmonary stenosis; $P D A$, patent ductus arteriosus; $N$, not performed; $R A A$, right aortic arch; $D S V C$, double superior venae cavae; $M P A$, main pulmonary artery; $R P A$, right pulmonary artery; $L P A$, left pulmonary artery; $M A P C A$, major aortopulmonary collateral artery (numbers in parentheses indicate numbers of MAPCAs); BTS, Blalock-Taussig shunt; $A P$, aorto-pulmonary; CoA, coarctation of aorta; $A R S A$, aberrant right subclavian artery; L-TGA, levo-transposition of the great arteries; TAPVR, total anomalous pulmonary venous return; ToF, tetralogy of Fallot; PAt, pulmonary atresia; $C+$, presence of pulmonary confluence; $C N$, confluence narrowing; $C-$, absence of pulmonary confluence; $M u$, multiple small aortopulmonary collateral arteries; PAPVR, partial anomalous pulmonary venous return; RAD, retroesophageal aortic diverticulum, ALSA, aberrant left subclavian artery; IVC, inferior vena cava. 
TABLE E2. Summary of aortic or venous developmental abnormalities and associated vascular abnormalities in 11 young patients with congenital heart diseases

\begin{tabular}{|c|c|c|c|c|c|}
\hline Case no. & Age/sex & Major diagnosis & Associated findings & MRA & $\begin{array}{l}\text { Catheterization/ } \\
\text { operation }\end{array}$ \\
\hline \multicolumn{6}{|c|}{ Coarctation of aorta } \\
\hline 1 & $24 \mathrm{mo} / \mathrm{M}$ & CoA (segmental) & PDA & 2 & $2 / \mathrm{N}$ \\
\hline 2 & $4 \mathrm{mo} / \mathrm{M}$ & CoA (segmental) & PDA, ARSA, DSVC & 4 & $4 / \mathrm{N}$ \\
\hline 3 & $11 \mathrm{mo} / \mathrm{F}$ & CoA (segmental) & PS, RAA, ALSA & 4 & $3 / \mathrm{N}$ \\
\hline 4 & $28 \mathrm{mo} / \mathrm{M}$ & CoA (segmental) & $\begin{array}{l}\text { Dilated LSA, multiple collaterals, right } \\
\text { inominate arterial occlusion, subclavian steal }\end{array}$ & 5 & $4 / \mathrm{N}$ \\
\hline 5 & $5 \mathrm{mo} / \mathrm{F}$ & CoA (hypoplastic arch) & Dilated LSA, multiple collaterals & 3 & $3 / 3$ \\
\hline 6 & $2 \mathrm{wk} / \mathrm{M}$ & CoA (hypoplastic arch) & Dilated LSA, multiple collaterals & 3 & $3 / 3$ \\
\hline 7 & $13 \mathrm{mo} / \mathrm{M}$ & CoA (hypoplastic arch) & Dilated LSA, multiple collaterals, DSVC & 4 & $4 / 4$ \\
\hline \multicolumn{6}{|c|}{ Anomalous aortic branching or veins } \\
\hline 8 & $27 \mathrm{mo} / \mathrm{M}$ & RAD, ARSA & & 2 & $2 / \mathrm{N}$ \\
\hline 9 & $1 \mathrm{wk} / \mathrm{M}$ & RAD, ARSA & & 2 & $2 / \mathrm{N}$ \\
\hline 10 & $36 \mathrm{mo} / \mathrm{F}$ & DSVC, LSVC to dilated coronary sinus & & 2 & $2 / \mathrm{N}$ \\
\hline 11 & $4 \mathrm{mo} / \mathrm{F}$ & Left isomerism & $\begin{array}{l}\text { Interrupted IVC, hemiazygos continuation, } \\
\text { DSVC, RAA, bilateral epbronchial PA }\end{array}$ & 5 & $4 / \mathrm{N}$ \\
\hline
\end{tabular}

MRA, Number of abnormalities seen on magnetic resonance angiography; Catheterization/operation, number of abnormalities seen on cardiac catheterization, surgical intervention, or both; $C O A$, coarctation of aorta; $P D A$, patent ductus arteriosus; $N$, not performed; $A R S A$, aberrant right subclavian artery; $D S V C$, double superior venae cavae; $P S$, pulmonary stenosis; $R A A$, right aortic arch; $A L S A$, aberrant left subclavian artery; $L S A$, left subclavian artery; $R A D$, retroesophageal aortic diverticulum; $A R S A$, aberrant right subclavian artery; $L S V C$, left superior vena cava; $I V C$, inferior vena cava; $P A$, pulmonary artery.

TABLE E3. Summary of pulmonary vascular developmental abnormalities and associated vascular abnormalities in 9 young patients with congenital heart diseases

\begin{tabular}{|c|c|c|c|c|c|c|}
\hline Case no. & Age/sex & Major diagnosis & Associated findings & Prior procedures & MRA & Catheterization/operation \\
\hline 1 & $27 \mathrm{mo} / \mathrm{F}$ & Pat & $\mathrm{C}+$, PDA, LPA aneurysm, DSVC & BTS & 4 & $\mathrm{~N} / 4$ \\
\hline 2 & $6 \mathrm{~d} / \mathrm{M}$ & Pat & $\mathrm{C}+, \mathrm{PDA}, \mathrm{RPA}$ stenosis & & 3 & $\mathrm{~N} / 3$ \\
\hline 3 & $40 \mathrm{mo} / \mathrm{M}$ & Pat & $\mathrm{C}+, \mathrm{PDA}$ & BTS & 2 & $2 / 2$ \\
\hline 4 & $7 \mathrm{mo} / \mathrm{M}$ & PS & ALSA & & 2 & $2 / \mathrm{N}$ \\
\hline 5 & $15 \mathrm{mo} / \mathrm{F}$ & PS & Widened PA, PDA & & 3 & $3 / \mathrm{N}$ \\
\hline 6 & $8 \mathrm{mo} / \mathrm{M}$ & PS & Widened PA, PDA, ALSA & & 4 & $4 / N$ \\
\hline 7 & $3 \mathrm{wk} / \mathrm{M}$ & $\begin{array}{l}\text { Widened PA with valve } \\
\text { dysplasia }\end{array}$ & PDA, RAA & & 3 & $4 / 4$ \\
\hline 8 & $6 \mathrm{mo} / \mathrm{M}$ & PAPVR & & & 1 & $1 / \mathrm{N}$ \\
\hline 9 & $4 d / F$ & TAPVR & PDA & & 2 & $\mathrm{~N} / 2$ \\
\hline
\end{tabular}

MRA, Number of abnormalities seen on magnetic resonance angiography; Catheterization/operation, number of abnormalities seen on cardiac catheterization, surgical intervention, or both; $P A t$, pulmonary atresia; $C+$, presence of pulmonary confluence; $P D A$, patent ductus arteriosus; $L P A$, left pulmonary artery; $D S V C$, double superior venae cavae; $B T S$, Blalock-Taussig shunt; $N$, not performed; RPA, right pulmonary artery; $P S$, pulmonary stenosis; $A L S A$, aberrant left subclavian artery; $P A$, pulmonary artery; $R A A$, right aortic arch; $P A P V R$, partial anomalous pulmonary venous return; $T A P V R$, total anomalous pulmonary venous return. 